\title{
Tissue Engineering of Small Caliber Vessel Grafts from Human Umbilical Veins
}

\author{
M.Hoenicka ${ }^{1}$, S. Schrammel' ${ }^{2}$, V.R. Jacobs ${ }^{3}$, G. Huber ${ }^{4}$, C. Schmid ${ }^{1}$ and D.E. Birnbaum ${ }^{1}$ \\ ${ }^{1}$ University of Regensburg Medical Center, Department of Cardiothoracic Surgery, Regensburg, Germany \\ ${ }^{2}$ University of Applied Sciences, FB Maschinenbau, Regensburg, Germany \\ ${ }^{3}$ Technical University Munich, Frauenklinik (OB/GYN), Munich, Germany \\ ${ }^{4}$ University of Regensburg, Krankenhaus Barmherzige Brüder, Klinik St. Hedwig, Regensburg, Germany
}

\begin{abstract}
Human umbilical veins (HUV) have recently been suggested as a starting material for vascular tissue engineering. HUV possess a functional smooth muscle layer and could be turned into an immunologically inert graft with contractile properties by creating a neoendothelium from the recipient's own cells. This study investigated methods to remove the native endothelium without impairing the contractile function of the smooth muscle layer. These denuded HUV were then seeded with endothelial cells in a perfusion bioreactor, demonstrating the creation of a confluent, shearresistant neoendothelium.
\end{abstract}

Keywords - vascular tissue engineering, endothelium, human umbilical vein, HUVEC, seeding

\section{INTRODUCTION}

Coronary artery disease is one of the most common maladies in western societies. Coronary artery bypass grafting with autologous vessels like internal mammary artery, saphenous vein, and radial artery has evolved into a routine procedure with satisfactory long-term results. However, there is a significant number of patients who cannot benefit from this procedure as they lack suitable autologous vessels as a consequence of varicosis, prior removal, or trauma.

Vessel grafts are supposed to match the size and the compliance of the host vessels. Synthetic materials have not met the expectations for small-caliber grafts due to compliance mismatches, infections, and thrombogenicity [1]. Biological scaffolds, especially those derived from vascular tissue, provide an excellent starting point for tissue-engineered small caliber vessel grafts. These scaffolds are obtained by removing existing cells by mechanical or chemical methods. Their biological surfaces promote vascular cell adhesion as they mostly consist of extracellular matrix. One potential problem is the immunogenicity of allogeneic or xenogeneic scaffolds even after decellularization.

Although glutaraldehyde-fixed human umbilical veins (HUV) have been used as grafts for peripheral reconstructions for decades [2], native HUV have been introduced as a starting material for vascular tissue engineering only re- cently. Daniel et al. used decellularized HUV [3], whereas our group suggested to keep the smooth muscle layer intact by using denuded HUV (denHUV) [4]. The immune properties of umbilical cord tissue are likely to permit allogeneic transplantation which was shown recently for umbilical cord stromal cells [5]. HUV were found to contract to serotonin and to U46619, to possess a biphasic stress-strain relationship [6], to retain part of the contractile function and of tissue viability after cryopreservation, and to release the antithrombotic agent prostacyclin even after denuding. Vascular function and antithrombotic properties were best in HUV from cesarean sections [7].

Although vascular grafts are supposed to carry endothelial cells harvested from the recipient, HUV endothelial cells (HUVEC) are a commonly used model in the design stage. Seeding of HUVEC onto denHUV has so far been demonstrated only on small, mechanically denuded patches under static conditions [6]. The current study identifies suitable methods to denude longer segments of HUV and to seed endothelial cells onto denHUV in a perfusion bioreactor.

\section{Materials AND Methods}

\section{Harvesting of umbilical cords}

Human umbilical cords from term or near-term pregnancies were stored in Krebs-Henseleit buffer as described previously [4]. Veins were dissected free from Wharton's Jelly and rinsed with phosphate-buffered saline.

\section{Denuding Methods}

HUV segments of $6-8 \mathrm{~cm}$ length were subjected to various denuding methods. Enzymatic denudation was performed according to a standard method of HUVEC isolation [8] using a collagenase $(0.1 \%)$ incubation of $20 \mathrm{~min}$ at $37^{\circ} \mathrm{C}$. Denudation by osmotic lysis was induced by perfusing HUV with distilled water for various times. Denudation by dehydration was performed by flushing HUV with carbogen ( $95 \%$ oxygen, $5 \%$ carbon dioxide) at $60 \mathrm{ml} / \mathrm{min}$ while 
the outside of the vessel was kept moist in culture medium [9]. After all denudation procedures the vessels were rinsed liberally with phosphate-buffered saline to protect the smooth muscle layer and to remove endothelial cell debris. In some experiments, HUV denuded by dehydration were filled with bovine gelatine $(0.25 \%)$, incubated for $30 \mathrm{~min}$ at $37^{\circ} \mathrm{C}$, and rinsed again. denHUV segments were then analyzed in an organ bath and by microscopy.

\section{Histology and scanning electron microscopy (SEM)}

Samples of denuded or seeded HUV were fixed in $4 \%$ formaldehyde and embedded in paraffin. Thin sections were stained by hematoxylin and eosin for bright field microscopy or stained with DAPI for fluorescence microscopy. Endothelial integrity was assessed visually by trained personnel. Samples for scanning electron microscopy were fixed in $2 \%$ formaldehyde and $2.5 \%$ glutardialdehyde, dehydrated, and sputtered with gold.

\section{Contractile properties}

Contractile functions of vessels were determined isometrically in an organ bath as described previously [4]. Maximum contractions caused by depolarization were determined by adding $150 \mathrm{mM} \mathrm{KCl}$ to the baths.

\section{Perfusion bioreactor}

denHUV were mounted in vessel chambers and attached to custom-built perfusion bioreactors operated at $37^{\circ} \mathrm{C}$. Each circulation consisted of medium reservoir, hollow fiber oxygenator $\left(20 \% \mathrm{O}_{2}, 5 \% \mathrm{CO}_{2}\right)$, perfusion and superfusion circuit pump hoses, and compliance chambers. Perfusion and superfusion circuits were driven by peristaltic pumps at $10 \mathrm{ml} / \mathrm{min}$ and $20 \mathrm{ml} / \mathrm{min}$, respectively. Ports at the vessel chamber inlets allowed to infuse cell suspensions for seeding. M199 with Earle's salts, supplemented with $20 \%$ fetal calf serum, penicillin, streptomycin, amphotericin $\mathrm{B}$, and gentamicin was used as culture medium.

\section{Seeding procedure}

HUVEC were obtained from HUV as described previously [4]. They were labelled with Ulex europaeus agglutinin (UEA) covalently linked to the fluorescent marker FITC. Cells were detached using trypsin/EDTA and resuspended in culture medium. Perfusion was stopped whereas superfusion continued to run, and cells were infused manually into the denHUV segments at a density of $4.5 \mathrm{E} 5 \mathrm{cells} / \mathrm{cm}^{2}$. The vessel chambers were rotated every other min for $10 \mathrm{~min}$ and every $5 \mathrm{~min}$ for another $50 \mathrm{~min}$ to enforce even seeding.
At $60,75,90$, and 105 min perfusion was turned on again briefly at $10 \mathrm{ml} / \mathrm{min}$. Starting at $120 \mathrm{~min}$ post seeding, the vessels were perfused at $10 \mathrm{ml} / \mathrm{min}$ for another $120 \mathrm{~min}$. Vessels were then fixed and analyzed by UV microscopy and SEM.

\section{RESULTS}

\section{Denudation}

Denudation by osmotic lysis successfully removed the endothelium if the incubation lasted 5 min or longer (Fig. 1 D-F). However, incubations of $3 \mathrm{~min}$ or longer significantly impaired contractions induced by $\mathrm{KCl}$ (Fig. 2).

Enzymatic treatment was also effective in removing the endothelium (Fig. 1 B). Microscopic analysis revealed extensive structural damage in the smooth muscle layer. Vessels treated with collagenase responded to $\mathrm{KCl}$ significantly weaker (Fig. 2).

Denudation by dehydration completely removed the endothelium (Fig. 1 C) without affecting contractile function (Fig. 3). Gelatine coating did not affect contractility of native HUV or of HUV denuded by dehydration $(\mathrm{p}=0.494$, ANOVA).

\section{Seeding}

Based on the results of the denudation experiments, only dehydration was used to denude vessels for seeding. As gelatine coating had no negative functional effects, some seeding experiments were done using gelatine coated denHUV.

Seeding with UEA-labelled HUVEC restored a confluent endothelium on denHUV. The en face SEM images show a smooth surface of the neoendothelium (Fig. 4 C). The cells appear larger but flatter compared to the native endothelium. The histological image of the cross section demonstrates by means of the fluorescence signal that the endothelial cells are indeed seeded cells (Fig. 4 D). Gelatin coating of denHUV had no visible effects on the seeding results (not shown).

\section{DISCUSSION}

This study compared denudation methods for HUV and demonstrated the seeding of a confluent neoendothelium on denuded HUV. 


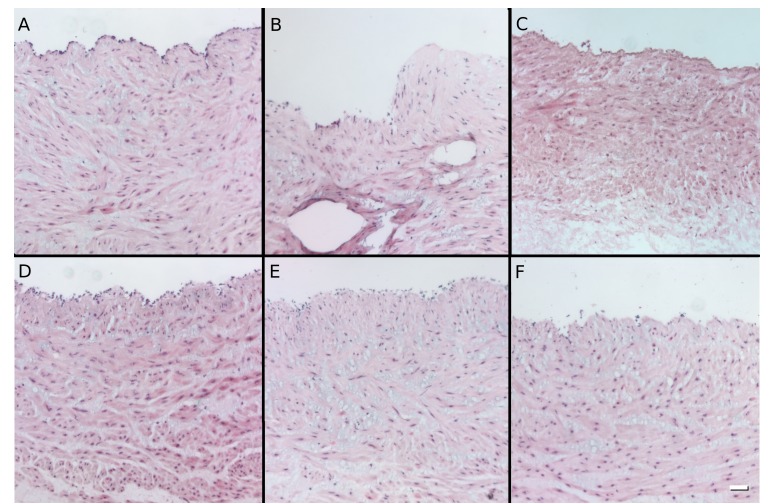

Fig 1: Cross sections of HUV stained with hematoxylin and eosin. (A) untreated control. (B) enzymatic denudation. (C) denuded by dehydration. (D-F) denudation by osmotic lysis for 1, 3, and 5 min, respectively. Bar indicates $50 \mu \mathrm{m}$.

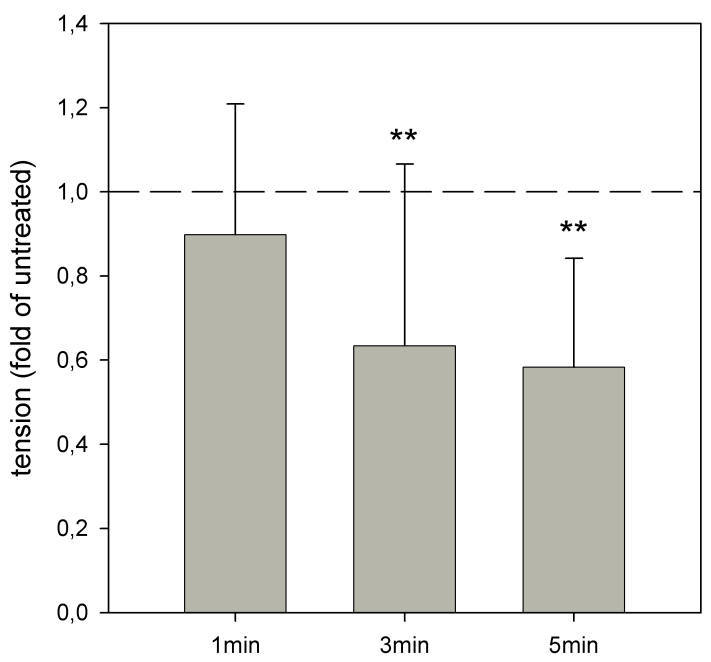

Fig 2: Contraction of HUV denuded by osmotic lysis. Vessels were challenged with $150 \mathrm{mM} \mathrm{KCl}$. Values are reported relative to untreated $\operatorname{HUV}(* * \mathrm{p}<0.01$, ANOVA)

As mentioned previously, one goal of this project was to reuse the existing smooth muscle layer of HUV to build the vessel walls of small caliber grafts. Therefore denudation methods had to be selected by their ability to keep this layer intact while removing the endothelium reliably at the same time. Previous static seeding experiments used mechanically denuded HUV [4]. This method is not well suited for long vessels and turned out to be detrimental to the contractile function. One particular feature of the vessel wall morphology is the presence of a basal lamina which separates the single layer of endothelial cells from the smooth muscle layer. This layer was hypothesized to protect the smooth muscle layer at least from some of the treatments required to remove the endothelial cells.

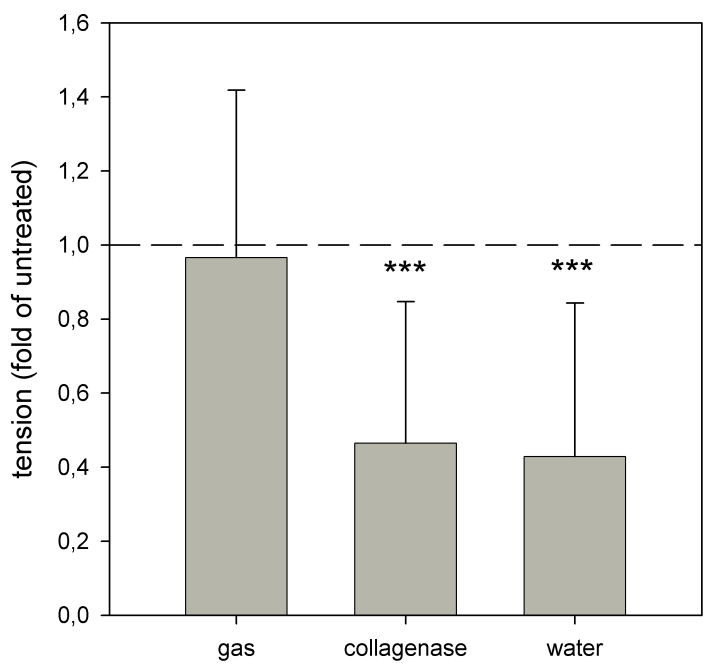

Fig 3: Comparison of denudation methods. Vessels were contracted with $150 \mathrm{mM} \mathrm{KCl}$. Values are reported relative to untreated vessels $(* * \mathrm{p}<0.01$, ANOVA)

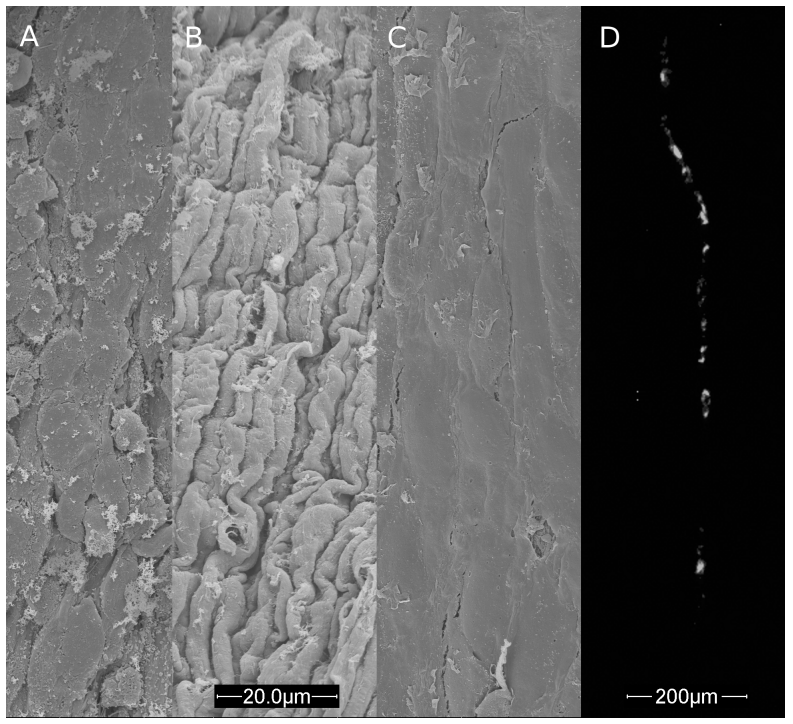

Fig 4: Seeding of denHUV with HUVEC. (A) SEM image of native HUV luminal surface. (B) SEM image of denHUV. (C) SEM image of seeded denHUV. (D) Fluorescence image of seeded denHUV cross section. Visible fluorescence indicates pre-labelled, seeded HUVEC.

Three denuding methods were identified and subjected to closer analysis. Enzymatic removal of endothelial cells is a common method to isolate and cultivate endothelial cells from umbilical veins and other vessels. Our data verified 
that endothelial cell removal is essentially complete under standard conditions. However, the smooth muscle layer was affected as well by the digestion, as it showed both substantial softening accompanied by structural damage and significantly reduced contractile function. Preliminary experiments ruled out shorter incubation times as this would leave parts of the endothelium intact. Enzymatic denudation therefore is not suitable to obtain denHUV for tissue engineering purposes.

Osmotic lysis turned out to be a highly effective method to disrupt and remove endothelial cells without inflicting visible damage to the smooth muscle layer. Incubation times of $5 \mathrm{~min}$ in the presence of distilled water were sufficient to remove the endothelum entirely. However, even in the absence of structural changes the smooth muscle layer was affected by this procedure. Contractile function declined at incubation times which were not sufficient to remove the endothelium completely.

Denuding by a stream of gas was originally developed to investigate a possible physiological role of the endothelium in microvessels. Although no mechanistic investigations are available, dehydration is likely to contribute a major part of the denuding action. After empirical optimization of flow rate and incubation time, this method appeared as a reliable and simple method to denude HUV. Moreover, contractile function was not affected at incubation times sufficient to remove the endothelium completely. Therefore this method was selected to prepare denHUV for seeding experiments.

Gelatin is a bovine product consisting mainly of hydrolyzed collagen. It resembles the extracellular matrix sufficiently to be used as a coating of cell culture flasks for endothelial cell culture. As the condition of the extracellular matrix after denudation was not known beforehand, it was prudent to test the hypothesis that gelatin coating improves cell adhesion during seeding. Gelatin coating did not affect the contractile functions of both HUV and denHUV and thus appeared safe to use.

The conditions required to obtain a uniform distribution of seeded endothelial cells on the luminal face of denHUV were arrived at empirically. Continuous rotation was not necessary. Instead, a protocol was used which involved rotation of the vessel for $90^{\circ}$ and $180^{\circ}$ in a fixed pattern to cover the entire surface. In contrast to seeding procedures of other scaffolds which may require static incubations ranging from 1 to $24 \mathrm{~h}$ [11], the first $30 \mathrm{~min}$ after infusing the cells appeared to be responsible for most if not all of the cell attachment. Uniform distribution was ascertained both by cross sections investigated for the fluorescent signal of the UEA-labelled cells and by SEM images of the entire luminal surface. HUV denuded by dehydration and seeded at a density of $4.5 \mathrm{E} 5$ cells $/ \mathrm{cm}^{2}$ regained a confluent and smooth endothelium using the described protocol. Coating denHUV with gelatin did not affect endothelial cell adhesion and was thus deemed unnecessary.

In conclusion, this study established a method to denude HUV without a loss of contractile function and without any noticeable limitation in vessel length. HUV denuded by this procedure is suitable for endothelial cell seeding in a perfusion system and shows an excellent cell adhesion, resulting in a confluent and shear-resistant endothelium within $4 \mathrm{~h}$ of seeding.

\section{ACKNOWLEDGMENT}

This study was supported by Deutsche Forschungsgemeinschaft (BI-139/2-1).

\section{REFERENCES}

1. Yow K, Ingram J, Korossis SA, et al. (2006) Tissue engineering of vascular conduits. Br J Surg 93:652-661

2. Dardik H, Wengerter K, Qin F, et al. (2002) Comparative decades of experience with glutaraldehyde-tanned human umbilical cord vein graft for lower limb revascularization: an analysis of 1275 cases. J Vasc Surg 35:64-71

3. Daniel J, Abe K, McFetridge PS (2005) Development of the human umbilical vein scaffold for cardiovascular tissue engineering applications. ASAIO J 51:252-261

4. Hoenicka M, Lehle K, Jacobs VR, et al. (2007) Properties of the human umbilical vein as a living scaffold for a tissue-engineered vessel graft. Tissue Eng 13:219-229

5. Weiss ML, Anderson C, Medicetty S, et al. (2008) Immune Properties of Human Umbilical Cord Wharton's Jelly-Derived Cells. Stem Cells 26:2865-2874

6. Hoenicka M, Lehle K, Jacobs VR, et al. (2007) Mechanical and seeding properties of human umbilical vein - a potential scaffold for a tissue-engineered vessel graft. Thorac Cardiovasc Surg 55:P_37

7. Hoenicka M, Jacobs VR, Huber G, et al. (2008) Advantages of human umbilical vein scaffolds derived from cesarean section vs. vaginal delivery for vascular tissue engineering. Biomaterials 29:1075-1084

8. Jaffe EA, Nachman RL, Becker CG, et al. (1973) Culture of human endothelial cells derived from umbilical veins. Identification by morphologic and immunologic criteria. J Clin Invest 52:2745-2756

9. Bjorling DE, Saban R, Tengowski MW, et al. (1992) Removal of venous endothelium with air. J Pharmacol Toxicol Methods 28:149-157

10. Alobaid N, Salacinski HJ, Sales KM, et al. (2005) Single stage cell seeding of small diameter prosthetic cardiovascular grafts. Clin Hemorheol Microcirc 33:209-226

Author: Markus Hoenicka, $\mathrm{PhD}$

Institute: University of Regensburg Medical Center, Department of Cardiothoracic Surgery

Street: Franz-Josef-Strauss-Allee 11

City: 93053 Regensburg

Country: Germany

Email: markus.hoenicka@klinik.uni-regensburg.de 